\title{
Nonoscillation, maximum principles, and exponential stability of second order delay differential equations without damping term
}

Alexander Domoshnitsky*

"Correspondence: adom@ariel.ac.il Ariel University, Ariel, Israel

\begin{abstract}
Delays, arising in nonoscillatory and stable ordinary differential equations, can induce oscillation and instability of their solutions. That is why the traditional direction in the study of nonoscillation and stability of delay equations is to establish a smallness of delay, allowing delay differential equations to preserve these convenient properties of ordinary differential equations with the same coefficients. In this paper, we find cases in which delays, arising in oscillatory and asymptotically unstable ordinary differential equations, induce nonoscillation and stability of delay equations. We demonstrate that, although the ordinary differential equation $x^{\prime \prime}(t)+c(t) x(t)=0$ can be oscillating and asymptotically unstable, the delay equation $x^{\prime \prime}(t)+a(t) x(t-h(t))-b(t) x(t-g(t))=$ 0 , where $c(t)=a(t)-b(t)$, can be nonoscillating and exponentially stable. Results on nonoscillation and exponential stability of delay differential equations are obtained. On the basis of these results on nonoscillation and stability, the new possibilities of non-invasive (non-evasive) control, which allow us to stabilize a motion of single mass point, are proposed. Stabilization of this sort, according to common belief, requires a damping term in the second order differential equation. Results obtained in this paper refute this delusion.
\end{abstract}

MSC: $34 \mathrm{~K} 20$

Keywords: nonoscillation; exponential stability; stabilization; maximum principles; Cauchy function; positivity of solutions; positivity of the Cauchy function; differential inequalities

\section{Introduction}

Let us start with one of motivations of this study considering a simplified model for motion of a single mass point

$$
X^{\prime \prime}(t)=f(t), \quad t \in[0,+\infty),
$$

where $X(t)=\operatorname{col}\left\{x_{1}(t), x_{2}(t), x_{3}(t)\right\}, f(t)=\operatorname{col}\left\{f_{1}(t), f_{2}(t), f_{3}(t)\right\}$. Let a solution $Y(t)=\operatorname{col}\left\{y_{1}(t)\right.$, $\left.y_{2}(t), y_{3}(t)\right\}$, which defines the trajectory we want to 'hold', be known and our purpose is to hold our object 'close' to this trajectory. It is impossible to 'achieve' this proximity on the semiaxis, because of instability of system (1.1). As a result, we have to make corrections of the motion changing the right-hand side $f$. We try to construct a control which makes this correction automatically. A standard approach is to construct a feedback con-

\section{Springer}

C2014 Domoshnitsky; licensee Springer. This is an Open Access article distributed under the terms of the Creative Commons Attribution License (http://creativecommons.org/licenses/by/2.0), which permits unrestricted use, distribution, and reproduction in any medium, provided the original work is properly cited. 
trol $u(t)$ which depends on the difference $X(t)-Y(t)$ or more exactly on the difference $X(t-\tau(t))-Y(t-\tau(t))$ since in real systems delay in receiving signal and in reaction on this signal arises. The control terms like

$$
u(t)=-\sum_{i=1}^{m} P_{i}(t)\left\{X\left(t-\tau_{i}(t)\right)-Y\left(t-\tau_{i}(t)\right)\right\}, \quad t \in[0,+\infty)
$$

(here $P_{i}(t)$ are $3 \times 3$ matrices, $i=1, \ldots, m$ ) are usually called non-invasive (non-evasive by other authors). Note that in laser physics the term 'Pyragas control' is widely used. Adding this control $u(t)$ into the equation of motion, we get

$$
X^{\prime \prime}(t)+\sum_{i=1}^{m} P_{i}(t) X\left(t-\tau_{i}(t)\right)=g(t), \quad t \in[0,+\infty),
$$

where

$$
g(t)=f(t)+\sum_{i=1}^{m} P_{i}(t) Y\left(t-\tau_{i}(t)\right), \quad t \in[0,+\infty)
$$

is the known right-hand side. Using the fact that $Y(t)$ satisfies $(1.1)$, i.e. $Y^{\prime \prime}(t)=f(t)$, and subtracting this equality from (1.3), we get the following system:

$$
Z^{\prime \prime}(t)+\sum_{i=1}^{m} P_{i}(t) Z\left(t-\tau_{i}(t)\right)=0, \quad t \in[0,+\infty)
$$

for the difference $Z(t)=X(t)-Y(t)$.

The problem of stabilization has been reduced to the exponential stability of this system. In the simplest case of diagonal matrices $P_{i}$, we get three separate equations. In the case of absence of delays $\left(\tau_{i}(t) \equiv 0, t \in[0,+\infty), i=1, \ldots, m\right)$ and constant diagonal entries, we get existence of positive roots of characteristic equation in the case of negative entries and pure imaginary roots in the case of positive ones. Thus, even in the case of the positive entries, each of these equations is Lyapunov stable, but not exponentially stable and, as a result, there is no stability with respect to the right-hand side (in the sense that small changes in the right-hand side could imply very essential changes in the solutions). In this paper we limit ourselves to diagonal matrices $P_{i}(t)(i=1, \ldots, m)$ in order to be concentrated on the scalar second order equation

$$
x^{\prime \prime}(t)+p x(t-\tau)=0, \quad t \in[0,+\infty)
$$

and its natural generalizations with variable coefficients and delays

$$
x^{\prime \prime}(t)+p(t) x(t-\tau(t))=0, \quad t \in[0,+\infty)
$$

and

$$
x^{\prime \prime}(t)+\sum_{i=1}^{m} p_{i}(t) x\left(t-\tau_{i}(t)\right)=0, \quad t \in[0,+\infty)
$$


where

$$
x(\xi)=0 \quad \text { for } \xi<0,
$$

$p_{i}$ and $\tau_{i}$ are measurable essentially bounded functions (below we write this as $p_{i} \in L_{\infty}$ ) $(i=1, \ldots, m)$.

We understand the solution of (1.7) in the traditional sense like in the book [1], i.e. as a function $x:[0,+\infty) \rightarrow(-\infty,+\infty)$ with absolutely continuous derivative $x^{\prime}$ on every finite interval such that $x^{\prime \prime} \in L_{\infty}$, satisfying (1.7).

Note that the delay equation (1.7) usually inherits the oscillation properties of the corresponding ODE

$$
x^{\prime \prime}(t)+p(t) x(t)=0 .
$$

For example, it was proven by Brands [2] that for every nonnegative $p(t)$ and bounded delay $\tau(t),(1.7)$ is oscillatory if and only if corresponding ordinary differential equation (1.10) is oscillatory. The asymptotic behavior of ODE (1.10) is not inherited by (1.7): Hale in his well-known book [3, Chapter 5, Section 5.4], discussed the characteristic equation

$$
\lambda^{2}+p e^{-\tau \lambda}=0
$$

for (1.6) and demonstrated that there exist at least two roots with positive real parts in the case $p \tau>\frac{\pi}{2}$. Myshkis, analyzing the roots of the characteristic equation (1.11), proved instability of equation (1.6) for each couple of positive constants $p$ and $\tau$ (see [4, Chapter III, Section 16, pp.105-106]). Note that the degree of instability of (1.6) (the maximum among positive real parts of the roots to (1.11)) tends to zero when $\tau \rightarrow$ 0 and $\tau \rightarrow+\infty$ [4]. The results on existence of unbounded solutions in the case of variable coefficients and delays were obtained on the basis of a growth of the Wronskians in [5] and developed in [6, 7]. One of the results can be formulated as follows [6]: if there exists a positive constant $\varepsilon$ such that $\tau(t)>\varepsilon$ and $p(t)>\varepsilon$, then there exist unbounded solutions to (1.7). In the paper [8] results on the instability of the system

$$
X^{\prime \prime}(t)+P(t) X(t-\omega)=0, \quad t \in(-\infty,+\infty)
$$

where $\omega>0$ and $P(t)$ is an $\omega$-periodic symmetric matrix function, were obtained.

Results on the boundedness of solutions to the delay equation (1.7) in the case of nondecreasing coefficient $p(t)$ and $\int_{0}^{\infty} p^{\alpha}(t) \tau(t) d t<\infty$ for a corresponding $\alpha$ were obtained in the paper [9]. The asymptotic formulas of solutions to the second order equation (1.6) are presented in [4, Chapter III, Section 16] and to (1.7) with a delay $\tau(t)$ summable on the semiaxis in $[10,11]$. In [6] it was found that all solutions of (1.7) with positive nondecreasing and bounded coefficient $p(t)$ and nondecreasing $h(t) \equiv t-\tau(t)$ are bounded if and only if

$$
\int_{0}^{\infty} \tau(t) d t<\infty
$$


Note that an approach for studying asymptotic properties of equations with linear transformations of arguments

$$
x^{\prime \prime}(t)=\sum_{j=-l, j \neq 0}^{l} a_{j} x\left(q^{j} t\right)+\lambda x(t), \quad t \in(-\infty,+\infty),
$$

where $a_{j}, q$ and $\lambda$ are constants, was proposed in [12] and developed in [13], where the equations with a combination of delayed and advanced arguments are considered. A study of advanced equations (see, for example, $(1.7)$, where $\tau(t) \leq 0)$ can be found in the paper [14] in which results on boundedness, stability, and asymptotic representations of solutions are obtained.

Summarizing, we can conclude that it appears to be impossible to obtain results as regards the exponential stability for one term of (1.7). It means that the control of the form

$$
u(t)=-P(t)\{X(t-\tau(t))-Y(t-\tau(t))\},
$$

without damping term cannot help us in the stabilization of system (1.1). Stability and instability of the second order delay differential equation

$$
x^{\prime \prime}(t)+q_{1} x^{\prime}(t)+q_{2} x^{\prime}(t-\tau)+p_{1} x(t)+p_{2} x(t-\tau)=0
$$

with constant coefficients was studied in $[15,16]$, where it was noted that this equation in the case $p_{1} p_{2}<0$ is of interest in machine tool analysis, in biology in explaining selfbalancing of the human body and in robotics in constructing biped robots (see the bibliography therein). Results on the stability of the equation

$$
x^{\prime \prime}(t)+q(t) x^{\prime}(t)+p(t) x(t-\tau(t))=0, \quad q>0, p>0,
$$

were obtained in $[17,18]$. First results on the exponential stability of the equation

$$
x^{\prime \prime}(t)+q(t) x^{\prime}(t-\theta(t))+p(t) x(t-\tau(t))=0, \quad q>0, p>0,
$$

without the assumption $\theta(t) \equiv 0$, as far as we know, were obtained in [19] and developed in $[20,21]$. The asymptotic stability of the equation

$$
x^{\prime \prime}(t)+p_{1} x(t)+p_{2} x(t-\tau)=0,
$$

with constant delay $\tau$ and coefficients $p_{1}$ and $p_{2}$ and without damping term were obtained in the paper [15]. These results were based on Pontryagin's technique for analysis of the roots of quasi-polynomials [22] and could not be used in the case of (1.8) with variable coefficients and/or delays. Note also that the assumption as regards the absence of the delay in the first term does not allow us to use this result of [15] in the stabilization in the model of the motion described in (1.1) by the delay control (1.2). Let us try to imagine situations in which variable delays and coefficients arising in the delayed feedback control may be important: (1) the case of control for missiles, where the delay depends on their distance from the controller and is variable; (2) spending of fuel implies the change of 
the mass of the missiles, which leads to variable coefficients in the delay system. As a conclusion, it should be stressed that there are no results for the exponential stability of the second order equation (1.8) without damping term in the case of variable coefficients and/or delays.

It will be demonstrated that the control of the form (1.2), where $m \geq 2$, can stabilize system (1.1). We obtain results as regards the exponential stability of (1.8), which are based on the maximum principles for the second order delay differential equation (1.8). Denoting $(M x)(t)=x^{\prime \prime}(t)+\sum_{i=1}^{m} p_{i}(t) x\left(t-\tau_{i}(t)\right)$, we can formulate the maximum inequalities principle [23] in the form: it follows from the inequalities $(M y)(t) \geq(M x)(t), y(0) \geq x(0)$, $y^{\prime}(0) \geq x^{\prime}(0)$ that $y(t) \geq x(t)$ for all $t \in[0, \infty)$. Using the solutions' representation (see (2.5) below), we can see that this principle is reduced to positivity of the Cauchy (fundamental) function of (1.8) and positivity of its nontrivial solution with nonnegative initial conditions. Nonoscillation of solutions in many cases leads to this property of the solution of second order equation (1.8). The positivity of the Cauchy function will open a way to the analysis of the asymptotic stability of nonlinear delay differential equations on the basis of the known schemes of upper and lower functions. The maximum boundedness principle [23] can be described in the form: there exists a constant $\Omega$ such that the solution of the initial value problem $(M x)(t)=f(t), x(0)=\alpha, x^{\prime}(0)=\beta$ satisfies the inequality $|x(t)| \leq \Omega(|f(t)|+|\alpha|+|\beta|)$ for $t \in[0, \infty)$. A generalization of the Perron theorem (see, for example, Theorem 3.5 in [1]) claims the exponential stability in this case.

The paper is organized as follows. After formulation of stabilization problem in Section 1, we discuss formulas of solution representations in Section 2. Simple results on stability of delay differential equations in the case $m=2$, which will be proven on the basis of general assertions obtained in Section 5, are formulated in Section 3. Auxiliary results used in the proof of our main assertions can be found in Section 4. Results on the positivity of the Cauchy function $C(t, s)$, nonoscillation and exponential stability are obtained in Sections 5 and 6. Discussion and open problems are presented in Section 7.

\section{About representation of solutions for second order delay differential equations}

The main object of this paper is the second order delay differential equation

$$
\begin{aligned}
& x^{\prime \prime}(t)+\sum_{i=1}^{m} p_{i}(t) x\left(t-\tau_{i}(t)\right)=f(t), \quad t \in[0,+\infty), \\
& x(\xi)=\varphi(\xi) \quad \text { for } \xi<0,
\end{aligned}
$$

with measurable essentially bounded $f, p_{i}, \varphi, \tau_{i}(i=0,1, \ldots, n-1)$, and $\tau_{i}(t) \geq 0$ for $t \geq 0$. In the traditional approach (see, for example, $[4,24,25]$ ), the homogeneous equation is considered as the equation

$$
x^{\prime \prime}(t)+\sum_{i=1}^{m} p_{i}(t) x\left(t-\tau_{i}(t)\right)=0, \quad t \in[0,+\infty),
$$

with the initial function (2.2). If (2.3), (2.2) are considered for all possible continuous functions $\varphi$, the space of its solutions is infinite-dimensional. In [26] a homogeneous equation 
was defined as (2.3) with the zero initial function

$$
x(\xi)=0 \quad \text { for } \xi<0 .
$$

This definition of the homogeneous equation allows researchers to build an analog of the classical general theory of ordinary differential equations (ODEs) for functional differential equations using the key notions of the classical ODEs theory, and it is based on the fact that the space of the solutions of the second order equation (2.3), (2.4) becomes two-dimensional. Note that (2.3), (2.2) can be written as (2.1), (2.4), where $f(t)=$ $-\sum_{i=1}^{m} p_{i}(t) \varphi\left(t-\tau_{i}(t)\right)\left\{1-\chi\left(t-\tau_{i}(t)\right)\right\}, \chi(t)=1$ for $t \geq 0$, and $\chi(t)=0$ for $t<0$. Thus (2.1), (2.4) is a nonhomogeneous one according to the approach of the book [1].

The general solution of (2.1), (2.4) (we understand the notion of the general solution exactly like in the theory of ODEs) can be represented in the form [26]

$$
x(t)=\int_{0}^{t} C(t, s) f(s) d s+x_{1}(t) x(0)+x_{2}(t) x^{\prime}(0),
$$

where $x_{1}(t), x_{2}(t)$ are two solutions of the homogeneous equation (2.3), (2.4) satisfying the conditions

$$
x_{1}(0)=1, \quad x_{1}^{\prime}(0)=0, \quad x_{2}(0)=0, \quad x_{2}^{\prime}(0)=1,
$$

and the kernel in this representation is called the Cauchy function $C(t, s)$ of $(2.1)$. Comparing this representation with the variation-of-constant formula of Hale [3, Chapter 6, Section 6.1], we see that they almost coincide; the kernel in the integral term is called the fundamental function by his numerous followers. For every fixed $s$ the function $C(t, s)$ as a function of the variable $t$ satisfies the equation $[1,26]$

$$
\begin{aligned}
& x^{\prime \prime}(t)+\sum_{i=1}^{m} p_{i}(t) x\left(t-\tau_{i}(t)\right)=0, \quad t \in[s,+\infty), \\
& x(\xi)=0 \quad \text { for } \xi<s,
\end{aligned}
$$

and the initial conditions $C(s, s)=0, C_{t}^{\prime}(s, s)=1$. Note that we will use this definition of the Cauchy function for the construction of $C(t, s)$ below. The behavior of the fundamental system $x_{1}, x_{2}$ of solutions of (2.3), (2.4) determines the existence and uniqueness of solutions of the boundary value problems for this equation. Positivity of the Cauchy function $C(t, s)$ of $(2.1),(2.4)$ is a basis of various theorems about differential inequalities (under corresponding conditions, the solution of an inequality is greater than the solution of an equation). These theorems help essentially in the study of stability for delay differential equations and their natural generalization - functional differential equations (FDEs) [1].

Let us formulate several definitions concerning stability.

Definition 2.1 Equation (2.3) is uniformly exponentially stable if there exist $N>0$ and $\alpha>0$, such that the solution of (2.3), (2.9), where

$$
x(\xi)=\varphi(\xi), \quad \xi<t_{0}, \quad x\left(t_{0}\right)=x_{0}, \quad x^{\prime}\left(t_{0}\right)=x_{0}^{\prime},
$$


satisfies the estimate

$$
|x(t)| \leq N e^{-\alpha\left(t-t_{0}\right)}, \quad t_{0} \leq t<+\infty
$$

where $N$ and $\alpha$ do not depend on $t_{0}$.

Definition 2.2 We say that the Cauchy function $C(t, s)$ of (2.1) satisfies the exponential estimate if there exist positive $N$ and $\alpha$ such that

$$
|C(t, s)| \leq N e^{-\alpha(t-s)}, \quad 0 \leq s \leq t<+\infty .
$$

It is well known that for (2.1) with bounded delays these two definitions are equivalent [1].

\section{Tests of positivity of the Cauchy functions and stability}

Let us try to understand in which direction nonoscillation of (1.8) can be established. Taking into account the well-known oscillation results [27], we come to the conclusion: only such smallness of the nonnegative coefficient $p(t)$ that its integral on the semiaxis is bounded may be suitable for nonoscillation of (1.7). In the case of (2.1) the analogous smallness of the sum of the nonnegative coefficients $p_{i}(t)$ should be required for nonoscillation. Regarding stability, we know that the smallness of the delay (1.13) is necessary and sufficient for boundedness of all solutions to (1.7) with constant coefficient $p(t) \equiv p>0$. It seems strange even to hope that assertions on nonoscillation and exponential stability can be achieved in the cases of non-tending to zero coefficients and delays. We will demonstrate that, although the ordinary differential equation

$$
x^{\prime \prime}(t)+\left\{\sum_{i=1}^{m} p_{i}(t)\right\} x(t)=0, \quad t \in[0,+\infty),
$$

can be oscillating and asymptotically unstable, the delay equation (2.3), (2.4) under corresponding conditions on the coefficients $p_{i}(t)$ and delays $\tau_{i}(t)$ is nonoscillating and exponentially stable. The basic idea of our approach is to avoid the condition on the nonnegativity of the coefficients $p_{i}(t)$ for all $i=1, \ldots, m$, and to allow terms with positive and terms with negative coefficients $p_{i}(t)$ to compensate each other.

In order to formulate several simple corollaries of the main results proven below in Section 5 , let us consider the equation

$$
\begin{aligned}
& x^{\prime \prime}(t)+a(t) x(t-\tau(t))-b(t) x(t-\theta(t))=0, \quad t \in[0,+\infty), \\
& x(\xi)=0 \quad \text { for } \xi<0,
\end{aligned}
$$

where $a(t), b(t), \tau(t)$, and $\theta(t)$ are measurable essentially bounded nonnegative functions. We denote

$$
q_{*}=\underset{t \geq 0}{\operatorname{essinf}} q(t), \quad q^{*}=\underset{t \geq 0}{\operatorname{esssup}} q(t) .
$$


Theorem 3.1 Assume that $0 \leq \tau(t) \leq \theta(t), 0 \leq b(t) \leq a(t)$,

$$
\begin{aligned}
& 4\{a(t)-b(t)\} \leq[b(\theta-\tau)]_{*}^{2}, \quad t \in[0,+\infty), \\
& 0<[b(\theta-\tau)]^{*} \theta^{*} \leq \frac{1}{e}
\end{aligned}
$$

Then

(1) the Cauchy function $C(t, s)$ of (3.2) is nonnegative for $0 \leq s<t<+\infty$;

(2) if there exists such positive $\varepsilon$ such that

$$
a(t)-b(t) \geq \varepsilon
$$

then the Cauchy function $C(t, s)$ of (3.2) satisfies the exponential estimate (2.11) and the integral estimate

$$
\sup _{t \geq 0} \int_{0}^{t} C(t, s) d s \leq \frac{1}{\varepsilon}
$$

(3) if there exists $\lim _{t \rightarrow \infty}\{a(t)-b(t)\}=k$, with $k>0$, then

$$
\lim _{t \rightarrow \infty} \int_{0}^{t} C(t, s) d s=\frac{1}{k} .
$$

Corollary 3.1 Assume that the delays $\tau(t) \equiv \tau, \theta(t) \equiv \theta$ are constants and

$$
\begin{aligned}
& 0<\varepsilon \leq 4\{a(t)-b(t)\} \leq b_{*}^{2}(\theta-\tau)^{2}, \quad t \in[0,+\infty), \\
& 0<b^{*}(\theta-\tau) \theta \leq \frac{1}{e}
\end{aligned}
$$

Then the assertions of Theorem 3.1 are true.

Consider the equation

$$
x^{\prime \prime}(t)+a(t) x(t-\tau)=f(t), \quad t \in[0,+\infty), a(t) \geq a_{*}>0 .
$$

There exist unbounded solutions of corresponding homogeneous equation $x^{\prime \prime}(t)+a(t) x(t-$ $\tau)=0, t \in[0,+\infty)$ (see, for example, [4, Chapter III, Section 16, p.106] in the case of constant $a$, and noted above in Section 1 sufficient conditions for existence of unbounded solutions from the paper [6]). Together with the fact of the oscillation [27] of all solutions this leads to chaos in behavior of solutions in the sense that small errors in a measurement of $x\left(t_{0}\right)$ and $x^{\prime}\left(t_{0}\right)$ can imply unpredictable changes in $x\left(t_{0}+\omega\right)$ and $x^{\prime}\left(t_{0}+\omega\right)$. Thus (3.12) is unstable. To stabilize its solution $x(t)$ to the given 'trajectory' $y(t)$ satisfying this equation, we choose the control in the form

$$
u(t)=b(t)[x(t-\theta)-y(t-\theta)]
$$

Subtracting the equality

$$
y^{\prime \prime}(t)+a(t) y(t-\tau)=f(t), \quad t \in[0,+\infty)
$$


from the equation

$$
x^{\prime \prime}(t)+a(t) x(t-\tau)=b(t)[x(t-\theta)-y(t-\theta)]+f(t), \quad t \in[0,+\infty),
$$

we get the equation

$$
z^{\prime \prime}(t)+a(t) z(t-\tau)-b(t) z(t-\theta)=0, \quad t \in[0,+\infty)
$$

for the difference $z(t)=x(t)-y(t)$. The stabilization has been reduced to the stability of this equation. A possible algorithm to construct this stabilizing control is clear now: first we choose the delay $\theta$ close to $\tau$ such that condition (3.11) is fulfilled, then we choose $b(t)$ close to $a(t)$ such that condition (3.10) is fulfilled.

Example 3.1 Stabilizing (3.12), where $a(t) \equiv a$, let us choose the control in the form (3.13) with constant coefficient $b(t) \equiv b$. We come to the study of the exponential stability of the equation

$$
x^{\prime \prime}(t)+a x(t-\tau)-b x(t-\theta)=g(t), \quad t \in[0,+\infty),
$$

with constant coefficient and delays and $g(t)=f(t)+b y(t-\theta)$. We can choose $\theta-\tau=\frac{1}{e b \theta}$ from (3.11); then from (3.10) we get the following condition of the exponential stability:

$$
0<4\{a-b\} \leq \frac{1}{e^{2} \theta^{2}} .
$$

Example 3.2 The equation

$$
x^{\prime \prime}(t)+a(t) x(t-\tau)=0, \quad a(t) \rightarrow+\infty, t \in[0,+\infty), \tau=\text { const },
$$

where $a(t) \geq a_{*}>0$, possesses oscillating solutions with amplitudes tending to infinity [6] that leads to the chaos in behavior of its solutions. This equation can also be stabilized by the control in the form (3.13). Consider, for example, the equation

$$
x^{\prime \prime}(t)+t x(t-\tau)=0, \quad t \in[1,+\infty), \tau=\text { const. }
$$

If we choose $b(t)=t-\Delta, \theta(t)=\tau+\frac{\gamma}{t}$, then the stabilization can be achieved by the control (3.13) with the parameters satisfying the inequalities

$$
0<2 \sqrt{\Delta}<\gamma<\frac{1}{\tau e} .
$$

In the following assertion we assume the smallness of the difference of the delays $\theta-\tau$ and do not assume the smallness of the delay $\theta$.

Consider for simplicity the equation

$$
\begin{aligned}
& x^{\prime \prime}(t)+a(t) x(t-\tau)-b(t) x(t-\theta)=0, \quad t \in[0,+\infty), \\
& x(\xi)=0 \text { for } \xi<0,
\end{aligned}
$$

with constant delays $\tau$ and $\theta$ and positive coefficients $a(t)$ and $b(t)$. 
Theorem 3.2 Assume that $0<\tau<\theta$, there exists a positive $\varepsilon$ such that

$$
\varepsilon \leq\{a(t)-b(t)\} \leq \frac{1}{4}[b(\theta-\tau)]_{*}^{2}, \quad t \in[0,+\infty)
$$

and

$$
\frac{1}{\sqrt{a^{*}} \exp \left\{\frac{b_{*}(\theta-\tau)^{2}}{4}\right\}} \operatorname{arctg} \frac{b_{*}(\theta-\tau)}{2 \sqrt{a^{*}} \exp \left\{\frac{b_{*}(\theta-\tau)^{2}}{4}\right\}}>\theta-\tau
$$

is fulfilled.

Then

(1) the Cauchy function $C(t, s)$ of (3.19) is nonnegative for $0 \leq s<t<+\infty$;

(2) the solutions $x_{1}(t), x_{2}(t)$ of (3.19), satisfying initial conditions (2.6), are nonegative for $0<t<+\infty$;

(3) the Cauchy function $C(t, s)$ of (3.19) satisfies the exponential estimate (2.11) and the integral estimate (3.8);

(4) if there exists $\lim _{t \rightarrow \infty}\{a(t)-b(t)\}=k$, then equality (3.9) is fulfilled.

Example 3.3 Consider the equation

$$
\begin{aligned}
& x^{\prime \prime}(t)+x(t)-b x(t-\theta)=0, \quad t \in[0,+\infty), \\
& x(\xi)=0, \quad \xi<0 .
\end{aligned}
$$

It is clear from the definition of the Cauchy function (see (2.7) and (2.8)) that for $t<\theta$, this equation is equivalent to the ordinary differential equation

$$
x^{\prime \prime}(t)+x(t)=0, \quad t \in[0, \theta]
$$

which Cauchy function is $C(t, s)=\sin (t-s)$. Let us demonstrate that condition (3.6) is essential for positivity of the Cauchy function $C(t, s)$ in Theorem 3.1, assuming that the numbers $b$ and $\theta$ are chosen such that all other conditions of Theorem 3.1 are fulfilled. If $\pi<\theta$, then in the triangle $0 \leq s \leq t<\theta$, its Cauchy function $C(t, s)=\sin (t-s)$ changes its sign. The same example demonstrates that condition (3.22) is essential in Theorem 3.2 for the positivity of $C(t, s)$. Note that in the case $\frac{\pi}{2}<\theta$, the solution $x_{1}(t)=\cos t$ satisfying conditions $(2.6)$ changes its sign on the interval $[0, \theta]$. It is clear that inequality (3.22) implies $\frac{\pi}{2}>\theta$.

Remark 3.1 Note the closely related results for the first order delay differential equation

$$
\begin{aligned}
& x^{\prime}(t)+a(t) x(t-\tau(t))-b(t) x(t-\theta(t))=0, \quad t \in[0,+\infty), \\
& x(\xi)=0, \quad \xi<0,
\end{aligned}
$$

which were obtained in [23]; see also Chapter 14 in the book [28]. Although the solutions of the equation

$$
x^{\prime}(t)+a(t) x(t-\tau(t))=0, \quad t \in[0,+\infty)
$$


can oscillate and the amplitudes of their solutions tend to infinity, the solutions of (3.25) cannot oscillate and tend to zero exponentially. For example, consider the equation

$$
x^{\prime}(t)+a x(t-\tau)=0, \quad a \tau>\frac{\pi}{2}, t \in[0,+\infty)
$$

with constant coefficient and delay, which has oscillating solutions with amplitudes tending to infinity, but the equation

$$
x^{\prime}(t)+a x(t-\tau)-b x(t-\theta)=0, \quad t \in[0,+\infty)
$$

is nonoscillating and exponentially stable if $b>0,0<(a-b) \theta \leq \frac{1}{e}, 0<a(\theta-\tau) \leq \frac{1}{e}$.

\section{Auxiliary results}

Let us formulate several results on systems of functional differential equations with Volterra operators. We mean the Volterra operators according to the following Tikhonov definition. Assume that $B: C_{[0, \infty)} \rightarrow L_{[0, \infty)}^{\infty}$ is a linear bounded operator, where $C_{[0, \infty)}$ and $L_{[0, \infty)}^{\infty}$ are the spaces of continuous and essentially bounded functions $x:[0,+\infty) \rightarrow$ $(-\infty,+\infty)$ respectively.

Definition 4.1 We say that an operator $B: C_{[0, \infty)} \rightarrow L_{[0, \infty)}^{\infty}$ is a Volterra one if for every two functions $y_{1}$ and $y_{2}$ and every $\omega$, the equality $y_{1}(t)=y_{2}(t)$ for $t \in[0, \omega]$ implies the equality $\left(B y_{1}\right)(t)=\left(B y_{2}\right)(t)$ for $t \in[0, \omega]$.

Consider the system

$$
\left(M_{i} x\right)(t) \equiv x_{i}^{\prime}(t)+\sum_{j=1}^{2}\left(B_{i j} x_{j}\right)(t)=f_{i}(t), \quad t \in[0,+\infty), i=1,2
$$

where $B_{i j}: C_{[0, \infty)} \rightarrow L_{[0, \infty)}^{\infty}$ are linear bounded Volterra operators for $i, j=1,2$. The general solution of this system can be represented in the form [1]

$$
x(t)=\int_{0}^{t} K(t, s) f(s) d s+K(t, 0) x(0)
$$

where $K(t, s)=\left\{K_{i j}(t, s)\right\}_{i, j=1,2}$ is called the Cauchy matrix of system (4.1), $x=\operatorname{col}\left\{x_{1}, x_{2}\right\}$, $f=\operatorname{col}\left\{f_{1}, f_{2}\right\}$.

Lemma 4.1 Let the Cauchy function $k(t, s)$ of the scalar equation

$$
x_{1}^{\prime}(t)+\left(B_{11} x_{1}\right)(t)=f_{1}(t), \quad t \in[0,+\infty),
$$

be positive in $0 \leq s \leq t<+\infty, B_{12}$ and $\left(-B_{21}\right)$ be positive operators. Then the following assertions are equivalent:

(1) $K_{21}(t, s) \geq 0$ and $K_{22}(t, s)>0$ for $0 \leq s \leq t<+\infty$;

(2) there exists a vector function $v=\operatorname{col}\left\{v_{1}, v_{2}\right\}$ with absolutely continuous components and essentially bounded derivatives $v^{\prime}=\operatorname{col}\left\{v_{1}^{\prime}, v_{2}^{\prime}\right\}$ such that

$$
v_{2}(t)>0, \quad\left(M_{i} v\right)(t) \leq 0, \quad i=1,2, t \in[0,+\infty), \quad v_{1}(0) \leq 0 .
$$


This lemma follows from Theorem 16.6 for the case $n=2$ (see [28, p.409]).

In order to formulate the conditions of positivity of the Cauchy function $k(t, s)$ of the scalar first order equation (4.3), let us introduce the function $H:[0,+\infty) \rightarrow[0,+\infty)$ which describes the 'size' of the memory of the operator $B_{11}$.

Definition 4.2 Let us define the function $H(t):[0,+\infty) \rightarrow[0,+\infty)$ as the maximal possible value on $[0, t]$ for which the equality $y_{1}(s)=y_{2}(s)$ for $s \in[H(t),+\infty)$ for every two continuous functions $y_{1}$ and $y_{2}:[0,+\infty) \rightarrow(-\infty,+\infty)$ implies the equality $\left(B_{11} y_{1}\right)(s)=$ $\left(B_{11} y_{2}\right)(s)$ for almost every $s \in[t,+\infty)$.

Lemma 4.2 Let $B_{11}: C_{[0, \infty)} \rightarrow L_{[0, \infty)}^{\infty}$ be a positive linear Volterra operator and

$$
\int_{H(t)}^{t}\left(B_{11} 1\right)(s) d s \leq \frac{1}{e}, \quad t \in(0,+\infty)
$$

then nontrivial solutions of the homogeneous equation

$$
x_{1}^{\prime}(t)+\left(B_{11} x_{1}\right)(t)=0, \quad t \in[0,+\infty)
$$

have no zeros for $t \in[0,+\infty)$ and its Cauchy function $C(t, s)>0$ for $0 \leq s \leq t<+\infty$.

This lemma follows from Theorem 15.7 (see [28, p.358]).

Denote $H^{*}=\operatorname{essup}_{t \geq 0}\{t-H(t)\}$.

Denoting $\left\|B_{11}\right\|$ the norm of the operator $B_{11}: C_{[0, \infty)} \rightarrow L_{[0, \infty)}^{\infty}$, as a corollary of this assertion we get the following.

Lemma 4.3 If $\left\|B_{11}\right\| H^{*} \leq \frac{1}{e}$, then the Cauchy function $C(t, s)$ of (4.3) is positive for $0 \leq s \leq$ $t<+\infty$.

Remark 4.1 Let us consider the second order scalar functional differential equation

$$
x^{\prime \prime}(t)+\left(B_{11} x^{\prime}\right)(t)+\left(B_{12} x\right)(t)=f_{1}(t), \quad t \in[0, \omega],
$$

and the corresponding system

$$
x_{1}^{\prime}(t)+\sum_{j=1}^{2}\left(B_{1 j} x_{j}\right)(t)=f_{1}(t), \quad x_{2}^{\prime}(t)-x_{1}(t)=0, \quad t \in[0, \omega] .
$$

The Cauchy function $C(t, s)$ of (4.7) coincides with the entry $K_{21}(t, s)$ of the Cauchy matrix $K(t, s)$ of system $(4.8)$ and $C_{t}^{\prime}(t, s)$ coincides with the entry $K_{11}(t, s)$. If the function $y(t)$ is the solution of the initial value problem

$$
\begin{aligned}
& x^{\prime \prime}(t)+\left(B_{11} x^{\prime}\right)(t)+\left(B_{12} x\right)(t)=0, \quad t \in[0, \omega], \\
& x(0)=1, \quad x^{\prime}(0)=0,
\end{aligned}
$$


then $K_{22}(t, 0)=y(t)$ and $K_{12}(t, 0)=y^{\prime}(t)$. Note that if $z(t)$ is the solution of the initial value problem consisting of equation (4.9) and the conditions

$$
x(0)=0, \quad x^{\prime}(0)=1,
$$

then $K_{21}(t, 0)=z(t), K_{11}(t, 0)=z^{\prime}(t)$.

\section{Main results}

Let us consider the equation

$$
(M x)(t) \equiv x^{\prime \prime}(t)+\sum_{i=1}^{2 m} p_{i}(t) x\left(t-\tau_{i}(t)\right)=f(t), \quad t \in[0,+\infty)
$$

and the corresponding homogeneous equation

$$
x^{\prime \prime}(t)+\sum_{i=1}^{2 m} p_{i}(t) x\left(t-\tau_{i}(t)\right)=0, \quad t \in[0,+\infty),
$$

where

$$
x(\xi)=0 \quad \text { for } \xi<0 .
$$

Denote

$$
\chi(t, s)= \begin{cases}1, & t \geq s \\ 0, & t<s\end{cases}
$$

Theorem 5.1 Let $(-1)^{i+1} p_{i}(t) \geq 0$ for $i=1, \ldots, 2 m, p_{2 i-1}(t)+p_{2 i}(t) \geq 0$ for $i=1, \ldots, m, t \in$ $[0,+\infty)$, and the Cauchy function of the first order equation

$$
y^{\prime}(t)+\sum_{i=1}^{m}\left|p_{2 i}(t)\right| \chi\left(t-\tau_{2 i}(t), 0\right) \int_{t-\tau_{2 i}(t)}^{t-\tau_{2 i-1}(t)} y(s) d s=0, \quad t \in[0,+\infty),
$$

be positive for $0 \leq s \leq t<+\infty$. Then the following assertions are equivalent:

(1) there exists a function $v$ with absolutely continuous and essentially bounded derivative $v^{\prime}$ and essentially bounded derivative $v^{\prime \prime}$ such that

$$
v(t)>0, \quad v^{\prime}(t) \leq 0, \quad(M v)(t) \leq 0, \quad t \in[0,+\infty) ;
$$

(2) there exists a bounded absolutely continuous function $u$ with essentially bounded derivative $u^{\prime}$ such that

$$
\begin{aligned}
& u(t) \geq 0, \\
& u^{2}(t)-u^{\prime}(t)+\sum_{i=1}^{2 m} p_{i}(t) \chi\left(t-\tau_{i}(t), 0\right) \exp \left\{\int_{t-\tau_{i}(t)}^{t} u(s) d s\right\} \leq 0, \quad t \in[0,+\infty)
\end{aligned}
$$


(3) the Cauchy function $C(t, s)$ of (5.1) is nonnegative for $0 \leq s<t<+\infty$, and solutions $x_{1}(t), x_{2}(t)$ of (5.2), satisfying initial conditions (2.6), are such that $x_{1}(t)>0, x_{2}(t) \geq 0$ for $0<t<+\infty$.

The proof of Theorem 5.1 is based on several auxiliary results.

Lemma 5.1 Assertions (1) and (2) of Theorem 5.1 are equivalent.

Proof $(2) \Rightarrow(1)$. In order to prove this implication, let us choose the function $v(t)$ in the condition (1) in the form

$$
v(t)=\exp \left\{-\int_{0}^{t} u(s) d s\right\}
$$

and demonstrate that it satisfies the condition (1). Differentiating, we get

$$
v^{\prime}(t)=-u(t) \exp \left\{-\int_{0}^{t} u(s) d s\right\}
$$

and

$$
v^{\prime \prime}(t)=\left[u^{2}(t)-u^{\prime}(t)\right] \exp \left\{-\int_{0}^{t} u(s) d s\right\} .
$$

We see that $v(t)>0, v^{\prime}(t) \leq 0$. In the calculation of $(M v)(t)$, one gets, carrying the exponent (5.6) out of the brackets,

$$
\begin{aligned}
(M v)(t)= & \exp \left\{-\int_{0}^{t} u(s) d s\right\} \\
& \times\left\{u^{2}(t)-u^{\prime}(t)+\sum_{i=1}^{2 m} p_{i}(t) \chi\left(t-\tau_{i}(t), 0\right) \exp \left[\int_{t-\tau_{i}(t)}^{t} u(s) d s\right]\right\} \leq 0 .
\end{aligned}
$$

It is clear now that inequalities (5.5) imply that $(M v)(t) \leq 0$. The implication $(2) \Rightarrow(1)$ has been proven.

$(1) \Rightarrow(2)$. Denote $u(t)=-\frac{v^{\prime}(t)}{v(t)}$, where the function $v(t)$ from the assertion (1), and demonstrate that this function satisfies the assertion (2). Obviously the function $u(t)$ is nonnegative. The function $v(t)$ satisfies the equation $v^{\prime}(t)+u(t) v(t)=0$ and consequently it can be represented in the form (5.6). The derivatives of the function $v(t)$ are defined by (5.7) and (5.8). From the inequality $(M v)(t) \leq 0$ we get inequality (5.9) and consequently condition (5.5) is fulfilled.

The implication $(1) \Rightarrow(2)$ has been proven.

Proof of Theorem 5.1 (1) $\Rightarrow$ (3). Let us rewrite (5.1) in the form

$$
\begin{aligned}
(M x)(t) \equiv & x^{\prime \prime}(t)+\sum_{i=1}^{m}\left|p_{2 i}(t)\right| \chi\left(t-\tau_{2 i}(t), 0\right)\left\{x\left(t-\tau_{2 i-1}(t)\right)-x\left(t-\tau_{2 i}(t)\right)\right\} \\
& +\sum_{i=1}^{m}\left|p_{2 i}(t)\right| x\left(t-\tau_{2 i-1}(t)\right) \chi\left(t-\tau_{2 i-1}(t), 0\right)\left(1-\chi\left(t-\tau_{2 i}(t), 0\right)\right)
\end{aligned}
$$




$$
\begin{aligned}
& +\sum_{i=1}^{m}\left[p_{2 i-1}(t)+p_{2 i}(t)\right] \chi\left(t-\tau_{2 i-1}(t), 0\right) x\left(t-\tau_{2 i-1}(t)\right) \\
= & f(t), \quad t \in[0,+\infty) .
\end{aligned}
$$

Consider the system

$$
\begin{aligned}
& x_{1}^{\prime}(t)+\sum_{i=1}^{m}\left|p_{2 i}(t)\right| \chi\left(t-\tau_{2 i}(t), 0\right) \int_{t-\tau_{2 i}(t)}^{t-\tau_{2 i-1}(t)} x_{1}(s) d s \\
& \quad+\sum_{i=1}^{m}\left|p_{2 i}(t)\right| x_{2}\left(t-\tau_{2 i-1}(t)\right) \chi\left(t-\tau_{2 i-1}(t), 0\right)\left(1-\chi\left(t-\tau_{2 i}(t), 0\right)\right) \\
& \quad+\sum_{i=1}^{m}\left[p_{2 i-1}(t)+p_{2 i}(t)\right] x_{2}\left(t-\tau_{2 i-1}(t)\right)=f(t), \\
& x_{2}^{\prime}(t)-x_{1}(t)=0, \quad t \in[0,+\infty),
\end{aligned}
$$

where

$$
x_{1}(\xi)=x_{2}(\xi)=0 \quad \text { for } \xi<0 .
$$

To compare this system with system (4.1), we observe that the operators $B_{i j}: C_{[0, \infty)} \rightarrow$ $L_{[0, \infty)}^{\infty}$ are of the following forms:

$$
\begin{aligned}
\left(B_{11} x_{1}\right)(t) \equiv & \sum_{i=1}^{m}\left|p_{2 i}(t)\right| \chi\left(t-\tau_{2 i}(t), 0\right) \int_{t-\tau_{2 i}(t)}^{t-\tau_{2 i-1}(t)} x_{1}(s) d s, \\
\left(B_{12} x_{2}\right)(t)= & \sum_{i=1}^{m}\left[p_{2 i-1}(t)+p_{2 i}(t)\right] \chi\left(t-\tau_{2 i-1}(t), 0\right) x_{2}\left(t-\tau_{2 i-1}(t)\right) \\
& +\sum_{i=1}^{m}\left|p_{2 i}(t)\right| x_{2}\left(t-\tau_{2 i-1}(t)\right) \chi\left(t-\tau_{2 i-1}(t), 0\right)\left(1-\chi\left(t-\tau_{2 i}(t), 0\right)\right), \\
\left(B_{21} x_{1}\right)(t)= & -x_{1}(t), \quad\left(B_{22} x_{2}\right)(t)=0 .
\end{aligned}
$$

Under the conditions $(-1)^{i+1} p_{i}(t) \geq 0, p_{2 i-1}(t)+p_{2 i}(t) \geq 0, \tau_{2 i-1}(t) \leq \tau_{2 i}(t)$ of Theorem 5.1, the operators $B_{11}, B_{12}$, and $\left(-B_{21}\right)$ are positive. Note that, according to the condition of Theorem 5.1, the Cauchy function of the first order equation (5.3) is positive for $0 \leq s \leq$ $t \leq+\infty$. The vector function $\operatorname{col}\left\{v_{1}(t), v_{2}(t)\right\}$, where $v_{1}(t)=v^{\prime}(t), v_{2}(t)=v(t)$, satisfies all conditions of assertion (2) of Lemma 4.1. According to Lemma 4.1, we get the positivity of the element $K_{22}(t, s)$ and the nonnegativity of the element $K_{21}(t, s)$ of the Cauchy matrix of system (5.11) for $0 \leq s \leq t \leq+\infty$. Remark 4.1 completes now the proof of the implication (1) $\Rightarrow(3)$.

To prove the implication (3) $\Rightarrow(1)$ we choose $v(t)=y_{1}(t)$, where $y_{1}(t)$ is the solution of the initial problem

$$
(M y)(t)=0, \quad y(0)=1, \quad y^{\prime}(0)=0 .
$$

Noting that the equivalence (1) $\Leftrightarrow(2)$ was obtained in Lemma 5.1, we can conclude that Theorem 5.1 has been proven. 
Denote $H^{*}=\operatorname{esssup}_{t \geq 0} \max _{1 \leq i \leq 2 m} \tau_{i}(t)$. For bounded $\tau_{i}(t)$ for $i=1, \ldots, m$, we have $H^{*}<$ $+\infty$.

Remark 5.1 The inequality

$$
\int_{t-H^{*}}^{t} \sum_{i=1}^{m}\left|p_{2 i}(s)\right|\left[\tau_{2 i}(s)-\tau_{2 i-1}(s)\right] d s \leq \frac{1}{e} \quad \text { for } t \geq 0
$$

where we set $p_{2 i}(s)=0$ for $s<0$, implies the positivity of the Cauchy function of the first order equation (5.3) (see Theorem 15.7, p.358 in [28]).

Remark 5.2 There is a corresponding inconvenience in choosing the functions $v(t)$ because of the equality $v\left(t-\tau_{i}(t)\right)=0$ for $t-\tau_{i}(t)<0$. Trying to avoid this inconvenience, we can make the following trick.

Let us define the auxiliary operator $B: C_{\left[-H^{*},+\infty\right)} \rightarrow L_{\left[-H^{*},+\infty\right)}^{\infty}$, where $C_{\left[-H^{*},+\infty\right)}$ and $L_{\left[-H^{*},+\infty\right)}^{\infty}$ are the spaces of continuous and of essentially bounded functions $x:\left[-H^{*}\right.$, $+\infty) \rightarrow(-\infty,+\infty)$, respectively, by the formula

$$
(B y)(t)= \begin{cases}\beta y(t), & -H^{*} \leq t \leq 0 \\ -\sum_{i=1}^{m} p_{2 i}(t) \int_{t-\tau_{2 i}(t)}^{t-\tau_{2 i-1}(t)} y(s) d s, & t \geq 0\end{cases}
$$

where the parameter $\beta$ will be defined below in the formulation of Theorem 5.2. Define the norm of this operator as $\|B\|=\max \left\{\beta\right.$, $\left.\operatorname{esssup}_{t \geq 0} \sum_{i=1}^{m}\left|p_{2 i}(t)\right|\left[\tau_{2 i}(t)-\tau_{2 i-1}(t)\right]\right\}$.

Let us define $p_{2 i-1}(t) \equiv 0, p_{2 i}(t) \equiv 0$ for $i=1, \ldots, m$ and $\tau_{j}(t) \equiv 0$ for $j=1, \ldots, 2 m$, on the interval $t \in\left[-H^{*}, 0\right)$, and consider the equation

$$
(M x)(t) \equiv x^{\prime \prime}(t)+q(t) x^{\prime}(t)+\sum_{i=1}^{2 m} p_{i}(t) x\left(t-\tau_{i}(t)\right)=f(t), \quad t \in\left[-H^{*},+\infty\right)
$$

where $f:\left[-H^{*},+\infty\right) \rightarrow(-\infty,+\infty)$ is a measurable essentially bounded function and

$$
q(t)= \begin{cases}\beta, & -H^{*} \leq t \leq 0 \\ 0, & t \geq 0\end{cases}
$$

with the same $\beta$ as in (5.18), which will be defined below.

It is clear from the definition of the Cauchy function by $(2.7),(2.8)$ that the Cauchy functions of (5.1) and (5.19) coincide in $0 \leq s \leq t<+\infty$.

Theorem 5.2 Assume that $(-1)^{i+1} p_{i}(t) \geq 0$ for $i=1, \ldots, 2 m, p_{2 i-1}(t)+p_{2 i}(t) \geq 0, \tau_{2 i-1}(t) \leq$ $\tau_{2 i}(t)$ for $i=1, \ldots, m$ and $t \in[0,+\infty)$ and there exists a real positive number $\alpha$ such that

(a) $\|B\| H^{*} \leq \frac{1}{e}$, where $\beta=\alpha$ in (5.18) defining the operator $B$;

(b) the inequality

$$
\alpha^{2}+\sum_{i=1}^{2 m} p_{i}(t) \exp \left\{\alpha \tau_{i}(t)\right\} \leq 0, \quad t \in[0,+\infty)
$$


Then

(1) the Cauchy function $C(t, s)$ of (5.19) is nonnegative for $0 \leq s<t<+\infty$;

(2) if in addition there exists a positive $\varepsilon$ such that

$$
\sum_{i=1}^{2 m} p_{i}(t) \geq \varepsilon
$$

then the Cauchy function $C(t, s)$ of $(5.1)$, its derivative $C_{t}^{\prime}(t, s)=K_{11}(t, s)$ and solutions of the homogeneous equation $M x=0$ satisfy the exponential estimate and the estimate

$$
\sup _{t \geq 0} \int_{0}^{t} C(t, s) d s \leq \frac{1}{\varepsilon}
$$

is true.

Proof of Theorem 5.2 Let us start with the proof of the assertion (1). We set $v(t)=$ $\exp \left\{-\alpha\left(t-H^{*}\right)\right\}$ for $t \in\left[-H^{*},+\infty\right)$ in the assertion (1) of the following reformulation of the assertion $(1) \Rightarrow(3)$ of Theorem 5.1 for (5.19).

Lemma 5.2 Let $(-1)^{i+1} p_{i}(t) \geq 0$ for $1=1, \ldots, 2 m, p_{2 i-1}(t)+p_{2 i}(t) \geq 0, \tau_{2 i-1}(t) \leq \tau_{2 i}(t)$ for $i=1, \ldots, m, t \in[0,+\infty)$ and the Cauchy function of the first order equation

$$
y^{\prime}(t)+(B y)(t)=f(t), \quad t \in\left[-H^{*},+\infty\right)
$$

where $\beta=\alpha$, be positive for $-H^{*} \leq s \leq t<+\infty$. Then the assertion (2) follows from the assertion (1), where

(1) there exists a function $v$ with absolutely continuous and essentially bounded derivative $v^{\prime}$ and essentially bounded derivative $v^{\prime \prime}$ such that

$$
v(t)>0, \quad v^{\prime}(t) \leq 0, \quad(M v)(t) \leq 0, \quad t \in\left[-H^{*},+\infty\right)
$$

(2) the Cauchy function $C(t, s)$ of (5.19) is nonnegative for $0 \leq s<t<+\infty$, and the solutions $x_{1}(t), x_{2}(t)$ of the homogeneous equation

$$
(M x)(t) \equiv x^{\prime \prime}(t)+q(t) x^{\prime}(t)+\sum_{i=1}^{2 m} p_{i}(t) x\left(t-\tau_{i}(t)\right)=0, \quad t \in\left[-H^{*},+\infty\right)
$$

satisfying the initial conditions (2.6), are such that $x_{1}(t)>0, x_{2}(t) \geq 0$ for $-H^{*}<t<+\infty$.

Substituting this function $v(t)=\exp \left\{-\alpha\left(t-H^{*}\right)\right\}$ into the differential operation $M$ defined by (5.19), we obtain the following two inequalities on $\left[-H^{*}, 0\right]$ and $[0,+\infty)$. The first one is

$$
\exp \left(-\alpha\left(t-H^{*}\right)\right)\left\{\alpha^{2}-\beta \alpha\right\} \leq 0, \quad t \in\left[-H^{*}, 0\right),
$$


and it is satisfied for $\beta=\alpha$. This explains how to choose $\beta$ in the definition of the operator $B$ by (5.18) and the coefficient $q(t)$ in (5.19). The second inequality is

$$
\alpha^{2} \exp (-\alpha t)+\sum_{i=1}^{2 m} p_{i}(t) \exp \left\{-\alpha\left(t-\tau_{i}(t)\right)\right\} \leq 0, \quad t \in[0,+\infty)
$$

and, after carrying the exponent out of the brackets, we get inequality (5.20). Thus inequalities (5.24) and (5.25) are results of a choice of the corresponding function $v(t)$ in assertion (1) of Lemma 5.2.

Equation (5.19) can be rewritten in the form

$$
(M x)(t) \equiv x^{\prime \prime}(t)+\left(B x^{\prime}\right)(t)+\sum_{i=1}^{2 m}\left\{p_{2 i-1}(t)+p_{2 i}(t)\right\} x\left(t-\tau_{i}(t)\right)=f(t), \quad t \in\left[-H^{*},+\infty\right),
$$

where the operator $B$ is defined by (5.18). The condition (a) implies, according to Lemma 4.3, the positivity of the Cauchy function of the first order equation (5.23).

Now we see that all conditions of Lemma 5.2 and assertion (1) are fulfilled. According to Lemma 5.2, we obtain assertion (1) of Theorem 5.2.

Let us prove assertion (2) of Theorem 5.2. The general solution of (5.19) can be represented in the form

$$
x(t)=\int_{-H^{*}}^{t} C(t, s) f(s) d s+x_{1}(t) x\left(-H^{*}\right)+x_{2}(t) x^{\prime}\left(-H^{*}\right),
$$

where solutions $x_{1}$ and $x_{2}$ of the homogeneous equation $(M x)(t)=0, t \in\left[-H^{*},+\infty\right)$ satisfy the conditions

$$
x_{1}\left(-H^{*}\right)=1, \quad x_{1}^{\prime}\left(-H^{*}\right)=0, \quad x_{2}\left(-H^{*}\right)=0, \quad x_{2}^{\prime}\left(-H^{*}\right)=1 .
$$

The function $y(t) \equiv \frac{1}{\varepsilon}$ satisfies the initial conditions

$$
x\left(-H^{*}\right)=\frac{1}{\varepsilon}, \quad x^{\prime}\left(-H^{*}\right)=0,
$$

and the equality

$$
\left(M \frac{1}{\varepsilon}\right)(t)=0, \quad t \in\left[-H^{*}, 0\right] .
$$

Using condition (5.21), we obtain

$$
\left(M \frac{1}{\varepsilon}\right)(t) \geq 1, \quad t \in[0,+\infty) .
$$

From representation (5.26), using (5.27)-(5.30), we get

$$
\frac{1}{\varepsilon}=\int_{0}^{t} C(t, s)\left(M \frac{1}{\varepsilon}\right)(s) d s+x_{1}(t) \frac{1}{\varepsilon}, \quad t \in[0,+\infty),
$$


and, taking into account the nonnegativity of $C(t, s)$, we get

$$
0 \leq \int_{0}^{t} C(t, s) d s+x_{1}(t) \frac{1}{\varepsilon} \leq \frac{1}{\varepsilon}, \quad t \in[0,+\infty) .
$$

Positivity of $x_{1}(t)$ and nonnegativity of $C(t, s)$ imply that

$$
\int_{0}^{t} C(t, s) d s \leq \frac{1}{\varepsilon}, \quad t \in[0,+\infty)
$$

this proves the integral estimate (5.22). It is clear that

$$
\int_{0}^{t} C(t, s)|f(s)| d s \leq \frac{1}{\varepsilon}, \quad t \in[0,+\infty)
$$

for every measurable essentially bounded $f$ such that $|f(t)| \leq 1$ for $t \in[0,+\infty)$. It means that the function

$$
y(t) \equiv \int_{0}^{t} C(t, s)|f(s)| d s, \quad t \in[0,+\infty)
$$

is bounded for every function $f$ measurable and bounded on the semiaxis $t \in[0,+\infty)$. Each of the solutions of (5.19) can be presented on $[0,+\infty)$ as a solution of the equation

$$
(M x)(t)=\psi(t), \quad t \in[0,+\infty),
$$

where $\psi(t)$ is a corresponding measurable bounded function such that $\psi(t)=f(t)$ for $t \geq$ $H^{*}$. Then the solution $x$ of (5.36) is bounded and consequently the solution of (5.19) is bounded for every measurable bounded function $f(t), t \in\left[-H^{*},+\infty\right)$ and

$$
\int_{-H^{*}}^{t} C(t, s)|f(s)| d s, \quad t \in[0,+\infty)
$$

is bounded for every measurable bounded function $f$.

Let us prove that the function $x_{2}(t)=C\left(t,-H^{*}\right)$ is bounded on the semiaxis $t \in\left[-H^{*}\right.$, $+\infty)$. The function $y(t)=1-\exp \left\{-t+H^{*}\right\}$ satisfies the Cauchy problem

$$
\begin{array}{ll}
(M x)(t)=f(t), & t \in\left[-H^{*},+\infty\right), \\
x\left(-H^{*}\right)=0, & x^{\prime}\left(-H^{*}\right)=1,
\end{array}
$$

where the differential operation $M$ defined by (5.19) and $f(t)=(M y)(t)$. It is clear that this function $f$ is bounded on the semiaxis $t \in\left[-H^{*},+\infty\right)$. The solution $y$ of (5.38), (5.39) can be written in the form

$$
1-\exp \left\{-t+H^{*}\right\}=\int_{-H^{*}}^{t} C(t, s) f(s) d s+x_{2}(t), \quad t \in\left[-H^{*},+\infty\right) .
$$

It follows now from the boundedness of the integral (5.37) for every bounded $f$ that $x_{2}(t)$ is bounded for $t \in\left[-H^{*},+\infty\right)$. 
Consider the system

$$
\left\{\begin{array}{l}
\left(M_{1} y\right)(t) \equiv y_{1}^{\prime}(t)+\left(B y_{1}\right)(t)+\sum_{i=1}^{m}\left\{p_{2 i-1}(t)+p_{2 i}(t)\right\} y_{2}\left(t-\tau_{2 i-1}(t)\right)=f_{1}(t) \\
\left(M_{2} y\right)(t) \equiv y_{2}^{\prime}(t)-y_{1}(t)=f_{2}(t), \quad t \in\left[-H^{*},+\infty\right)
\end{array}\right.
$$

If $\alpha$ satisfies inequality (5.20), the functions $v_{1}(t)=-\alpha \exp \left\{-\alpha\left(t+H^{*}\right)\right\}, v_{2}(t)=\exp \{-\alpha(t+$ $\left.H^{*}\right)$ \} satisfy the assertion (2) of Lemma 4.1. The condition (a) implies that all other conditions of Lemma 4.1 are also fulfilled. Now, according to Lemma 4.1, the entries in the second row of the Cauchy matrix $K(t, s)=\left\{K_{i j}(t, s)\right\}_{i, j=1}^{2}$ of system (5.41) satisfy the inequalities $K_{21}(t, s) \geq 0, K_{22}(t, s)>0$ for $0 \leq s \leq t<+\infty$. Let us substitute the constant vector $z=\operatorname{col}\left\{z_{1}, z_{2}\right\}$, where $z_{1}=-1$ and $z_{2}=\frac{1+\|B\|}{\varepsilon}$ instead of $y_{1}(t)$ and $y_{2}(t)$, respectively, into system (5.41). Condition (5.21) implies that $\left(M_{1} y\right)(t) \geq 1,\left(M_{2} y\right)(t)=1$. Consider now system (5.41), where $f_{1}(t) \equiv\left(M_{1} z\right)(t), f_{2}(t) \equiv 1$ for $t \in\left[-H^{*},+\infty\right)$. From representation (4.2) of the solution we get for the second component of the solution vector

$$
z_{2}=\int_{-H^{*}}^{t} K_{21}(t, s) f_{1}(s) d s+\int_{-H^{*}}^{t} K_{22}(t, s) d s+K_{22}(t,-H) \frac{1+\|B\|}{\varepsilon}-K_{21}\left(t,-H^{*}\right) .
$$

In Remark 4.1 we explained that $K_{21}(t, s)=C(t, s), K_{21}\left(t,-H^{*}\right)=x_{2}(t), K_{22}\left(t,-H^{*}\right)=x_{1}(t)$, where $C(t, s)$ is the Cauchy function and $x_{1}, x_{2}$ are solutions of the equation

$$
(M x)(t) \equiv x^{\prime \prime}(t)+q(t) x^{\prime}(t)+\sum_{i=1}^{m} p_{i}(t) x\left(t-\tau_{i}(t)\right)=0, \quad t \in\left[-H^{*},+\infty\right)
$$

satisfying conditions (5.27). Now it follows from the boundedness of the integral $\int_{-H^{*}}^{t} K_{21}(t$, $s) d s$ and solutions $x_{1}, x_{2}$ that the integral $\int_{-H^{*}}^{t} K_{22}(t, s) d s$ is bounded, and, using the positivity of $K_{22}(t, s)$, we can claim that the integral $\int_{-H^{*}}^{t} K_{22}(t, s) f(s) d s$ is bounded for every measurable bounded $f(t), t \in\left[-H^{*},+\infty\right)$. We see that the component $y_{2}$ of the solution vector to system (5.41) is bounded for every measurable bounded $f_{1}$ and $f_{2}$. The positivity of the Cauchy function of the first order equation (5.25) together with condition (5.21) imply the boundedness of the component $y_{1}$ of solution vector to system (5.41). Now a generalization of the Perron theorem (see, for example, Theorem 3.5 in [1]) claims that all entries of the Cauchy matrix $K(t, s)=\left\{K_{i j}(t, s)\right\}_{i, j=1}^{2}$ satisfy the exponential estimate (2.11). Each of the solutions of (5.1) coincides with a corresponding solution of (5.19) with the measurable essentially bounded right-hand side $\psi(t)$ such that $\psi(t)=f(t)$ for $t \geq H^{*}$. Thus for every bounded right-hand side, the solution of (5.1) is bounded, and the exponential estimate (2.11) of the Cauchy function of (5.1), its derivative $C_{t}^{\prime}(t, s)=K_{11}(t, s)$, and the solutions of the homogeneous equation $M x=0$ satisfy the exponential estimate (2.11).

Corollary 5.1 If under the conditions of Theorem 5.2 there exists a positive limit

$$
\lim _{t \rightarrow \infty} \sum_{i=1}^{2 m} p_{i}(t)=k
$$

then

$$
\lim _{t \rightarrow \infty} \int_{0}^{t} C(t, s) d s=\frac{1}{k}
$$


The proof of Corollary 5.1 can be obtained from equality (5.31) and the fact of the exponential estimate of all elements of the Cauchy matrix of system (5.41).

Remark 5.3 The positivity of $\varepsilon$ in condition (5.21) is essential, as the following example demonstrates. Consider the equation

$$
x^{\prime \prime}(t)+x\left(t-\left|\sin \frac{1}{2} t\right|\right)-x\left(t-2\left|\sin \frac{1}{2} t\right|\right)=0, \quad t \in[0,+\infty) .
$$

Here $\varepsilon=0$ and one of solutions is a constant and does not tend to zero when $t \rightarrow+\infty$.

Consider the equation

$$
(M x)(t) \equiv x^{\prime \prime}(t)+\sum_{i=1}^{2 m} p_{i}(t) x\left(t-\tau_{i}(t)\right)+\sum_{j=1}^{n} q_{j}(t) x\left(t-\theta_{j}(t)\right)=f(t), \quad t \in[0,+\infty),
$$

and the corresponding homogeneous equation

$$
x^{\prime \prime}(t)+\sum_{i=1}^{2 m} p_{i}(t) x\left(t-\tau_{i}(t)\right)+\sum_{j=1}^{n} q_{j}(t) x\left(t-\theta_{j}(t)\right)=0, \quad t \in[0,+\infty),
$$

where

$$
x(\xi)=0 \quad \text { for } \xi<0 \text {; }
$$

$q_{j}, \theta_{j}, p_{i}$ and $\tau_{i}$ are measurable essentially bounded functions for $j=1, \ldots, n, i=1, \ldots, m$.

Theorem 5.3 Let all assumptions of Theorem 5.2 be fulfilled.

Then

(1) if $q_{j}(t) \leq 0$ for $t \in[0,+\infty)$, then the Cauchy function $C(t, s)$ of $(5.47)$ is positive for $0 \leq s<t<+\infty$;

(2) if there exist positive $\varepsilon_{0}$ and $\varepsilon$ such that

$$
\sum_{i=1}^{2 m} p_{i}(t) \geq \varepsilon, \quad \varepsilon-\varepsilon_{0} \geq \sum_{j=1}^{n}\left|q_{j}(t)\right|, \quad t \in[0,+\infty),
$$

then the Cauchy function $C(t, s)$ of (5.47) satisfies the exponential estimate (2.11) and the integral estimate

$$
\sup _{t \geq 0} \int_{0}^{t}|C(t, s)| d s \leq \frac{1}{\varepsilon_{0}}
$$

Proof Using the formula of the solutions' representation (2.5), we can conclude that the solution of the initial problem (5.47), (5.49), (5.52), where

$$
x(0)=0, \quad x^{\prime}(0)=0,
$$

satisfies the integral equation

$$
x(t)=(K x)(t)+\psi(t),
$$


and the operator $K: C_{[0, \infty)} \rightarrow C_{[0, \infty)}$ is defined by equalities (5.54), (5.49), where

$$
(K x)(t)=-\int_{0}^{t} W(t, s) \sum_{j=1}^{n} q_{j}(s) x\left(s-\theta_{i}(s)\right) d s,
$$

where $W(t, s)$ is the Cauchy function of (5.1), and

$$
\psi(t)=\int_{0}^{t} W(t, s) f(s) d s
$$

According to Theorem 5.2, we obtain the positivity of $W(t, s)$. The operator $K$ in the case of nonpositive $q_{j}(t)$ is positive. If we consider this operator $K$ on every finite interval $[0, \omega]$ its spectral radius $\rho(K)$ is zero, and one can write

$$
x(t)=(I-K)^{-1} \psi(t)=\psi(t)+(K \psi)(t)+\left(K^{2} \psi\right)(t)+\cdots .
$$

If $f \geq 0$, then $\psi \geq 0$ and consequently $x \geq 0$. Reference to (2.5) completes the proof of the assertion (1).

In order to prove the assertion (2), we have to demonstrate that the solution $x$ is bounded for every bounded right-hand side $f$ and then the extension of the Bohl-Perron implies the exponential estimate (2.11) of the Cauchy matrix (see Theorem 3.5 in the book [1]). According to Theorem 5.2, the Cauchy function $W(t, s)$ of (5.1) satisfies the exponential estimate (2.11). This implies the boundedness of $\psi$ for every bounded $f$. If the norm of the operator $K: C_{[0, \infty)} \rightarrow C_{[0, \infty)}$ is less than one, then the bounded operator $(I-K)^{-1}$ exists. We have only to prove that $\|K\|<1$.

All conditions of Theorem 5.2 are fulfilled. According to Theorem 5.2, we have

$$
\|W\| \equiv \sup _{t \geq 0} \int_{0}^{t} W(t, s) d s \leq \frac{1}{\varepsilon}
$$

and

$$
\|K\| \leq\|W\| \underset{t \geq 0}{\operatorname{esssup}} \sum_{j=1}^{n}\left|q_{j}(t)\right| \leq \frac{1}{\varepsilon} \underset{t \geq 0}{\operatorname{essup}} \sum_{j=1}^{n}\left|q_{j}(t)\right| \leq \frac{\varepsilon-\varepsilon_{0}}{\varepsilon}<1 .
$$

This, according to our observations above, completes the proof of exponential estimate (2.11) of the Cauchy function of (5.47).

Now let us prove estimate (5.51). For the solution $x$ of the initial problem (5.47), (5.49), (5.52), we have two representations

$$
x(t)=\int_{0}^{t} C(t, s) f(s) d s,
$$

and (5.56). Estimating for $|f(t)| \leq 1$, we obtain

$$
|x(t)|=\left|\int_{0}^{t} C(t, s) f(s) d s\right| \leq\left\|(I-K)^{-1}\right\|\|W\| \leq \frac{1}{1-\frac{\varepsilon-\varepsilon_{0}}{\varepsilon}} \frac{1}{\varepsilon}=\frac{1}{\varepsilon_{0}} .
$$

This completes the proof of the assertion (2) of Theorem 5.3. 


\section{Proofs of Theorems 3.1 and 3.2}

Proof of Theorem 3.1 For (3.2), inequality (5.20) is of the following form:

$$
\alpha^{2}+a(t) \exp \{\alpha \tau(t)\}-b(t) \exp \{\alpha \theta(t)\} \leq 0, \quad t \in[0,+\infty),
$$

which can be written as

$$
\begin{aligned}
& \alpha^{2}+b(t)\{\exp [\alpha \tau(t)]-\exp [\alpha \theta(t)]\}+(a(t)-b(t)) \exp \{\alpha \tau(t)\} \\
& \quad \leq 0, \quad t \in[0,+\infty),
\end{aligned}
$$

or, using the Lagrange theorem,

$$
\begin{aligned}
& \alpha^{2}-\alpha b(t)(\theta(t)-\tau(t)) \exp [\alpha \eta(t)]+(a(t)-b(t)) \exp \{\alpha \tau(t)\} \\
& \quad \leq 0, \quad t \in[0,+\infty),
\end{aligned}
$$

where $\tau(t) \leq \eta(t) \leq \theta(t)$. Setting $\alpha=\frac{1}{2}\{b(t)(\theta(t)-\tau(t))\}_{*}$ we get

$$
\begin{aligned}
& \frac{1}{4}\{b(t)(\theta(t)-\tau(t))\}_{*}^{2}-\frac{1}{2}\{b(t)(\theta(t)-\tau(t))\}_{*} b(t)(\theta(t)-\tau(t)) \\
& \quad \times \exp \left[\frac{1}{2}\{b(t)(\theta(t)-\tau(t))\}_{*} \eta(t)\right] \\
& +(a(t)-b(t)) \exp \left\{\frac{1}{2}[b(t)(\theta(t)-\tau(t))]_{*} \tau(t)\right\} \leq 0, \quad t \in[0,+\infty), \\
& (a(t)-b(t)) \exp \left\{\frac{1}{2}[b(t)(\theta(t)-\tau(t))]_{*} \tau(t)\right\} \\
& \leq \frac{1}{4}\{b(t)(\theta(t)-\tau(t))\}_{*} b(t)(\theta(t)-\tau(t)) \\
& \quad \times \exp \left\{\frac{1}{2}[b(t)(\theta(t)-\tau(t))]_{*} \eta(t)\right\}, \quad t \in[0,+\infty),
\end{aligned}
$$

which is fulfilled if

$$
4(a(t)-b(t)) \leq\{b(t)(\theta(t)-\tau(t))\}_{*^{\prime}}^{2} \quad t \in[0,+\infty) .
$$

The inequality in the condition (a) of Theorem 5.2 is fulfilled if inequality (3.6) is satisfied.

Now Theorem 3.1 follows from Theorem 5.2 and Corollary 5.1.

Remark 6.1 We have not yet used the assertion (2) of Theorem 5.1 to obtain sufficient conditions for the positivity of the Cauchy function $C(t, s)$ of (3.2). Let us build the function $u(t)$, proving the following assertion.

Proof of Theorem 3.2 To construct the function $u(t)$ on the interval $[0, \tau]$ we solve the equation $u^{\prime}(t)=u^{2}(t)$. Its solution is

$$
u(t)=\frac{1}{c_{1}-t},
$$


where $c_{1}$ is a constant. We will explain below how to choose $c_{1}$. On the interval $[\tau, \theta]$ we solve the inequality

$$
u^{\prime}(t)=u^{2}(t)+a^{*} \exp \left\{\frac{b_{*}(\theta-\tau)^{2}}{2}\right\} .
$$

Separating variables and integrating, one gets

$$
\operatorname{arctg} \frac{u}{\sqrt{a^{*}} \exp \left\{\frac{b_{*}(\theta-\tau)^{2}}{4}\right\}}=\sqrt{a^{*}} \exp \left\{\frac{b_{*}(\theta-\tau)^{2}}{4}\right\}\left(t+c_{2}\right),
$$

where $c_{2}$ is a constant, and

$$
u(t)=\sqrt{a^{*}} \exp \left\{\frac{b_{*}(\theta-\tau)^{2}}{4}\right\} \operatorname{tg}\left[\sqrt{a^{*}} \exp \left\{\frac{b_{*}(\theta-\tau)^{2}}{4}\right\}\left(t+c_{2}\right)\right] .
$$

Choosing $c_{2}$ such that

$$
u(\theta)=\frac{b_{*}(\theta-\tau)}{2},
$$

we obtain

$$
c_{2}=\frac{1}{\sqrt{a^{*}} \exp \left\{\frac{b_{*}(\theta-\tau)^{2}}{4}\right\}} \operatorname{arctg} \frac{b_{*}(\theta-\tau)}{2 \sqrt{a^{*}} \exp \left\{\frac{b_{*}(\theta-\tau)^{2}}{4}\right\}}-\theta .
$$

For positivity of the function $u(t)$ for $t \in[\tau, \theta]$, we have to assume that $c_{2}>-\tau$, which follows from inequality (3.22).

Let us now choose the constant $c_{1}$ in (6.7) such that

$$
u(\tau)=\frac{1}{c_{1}-\tau},
$$

where $u(\tau)$ is defined by (6.10), (6.12).

Setting

$$
u(t) \equiv \frac{1}{2} b_{*}(\theta-\tau) \quad \text { for } t \geq \theta,
$$

we have built the positive absolutely continuous function $u(t)$ satisfying the condition (2) of Theorem 5.1. Now reference to Theorem 5.1 completes the proof of the assertions (1) and (2) of Theorem 3.2. To prove assertions (3) and (4) we actually repeat the proof of the assertion (2) of Theorem 5.2 (in the case $H^{*}=0$ ).

The proof of Theorem 3.2 have been completed.

\section{Open problems}

Let us formulate a list of problems to be solved in the future.

1. To obtain results on the exponential stability of the equation

$$
x^{\prime \prime}(t)+\sum_{j=1}^{n} q_{j}(t) x^{\prime}\left(t-\theta_{j}(t)\right)+\sum_{i=1}^{m} p_{i}(t) x\left(t-\tau_{i}(t)\right)=f(t), \quad t \in[0,+\infty),
$$


where $q_{j}(t) \leq 0$ for $t \in[0,+\infty)$. Results of this type were considered impossible. It was assumed in the previous works $[17-21]$ that $q_{j}(t)>0$, and in the presented paper $q_{j}(t) \equiv 0$ for $t \in[0,+\infty), j=1, \ldots, m$.

2. To obtain results as regards stabilization of the equation $x^{(n)}(t)=f(t)$, where $n>2$, to the trajectory $y(t)$ by the control of the form

$$
u(t)=-\sum_{i=1}^{m} p_{i}(t)\left\{x\left(t-\tau_{i}(t)\right)-y\left(t-\tau_{i}(t)\right)\right\}, \quad t \in[0,+\infty),
$$

without derivatives, i.e. to obtain results about the exponential stability of the equation

$$
x^{(n)}(t)+\sum_{i=1}^{m} p_{i}(t) x\left(t-\tau_{i}(t)\right)=f(t), \quad t \in[0,+\infty)
$$

Results of this type were considered impossible.

3. To obtain results on oscillation/nonoscillation, existence of solutions tending to zero or tending to infinity for the second order equation (2.3) without the assumption of the nonnegativity of the coefficients.

4. To obtain results on stabilization of system (1.1) to the trajectory $Y(t)$ by the control of the form

$$
u(t)=-\sum_{i=1}^{m} P_{i}(t)\left\{X\left(t-\tau_{i}(t)\right)-Y\left(t-\tau_{i}(t)\right)\right\}, \quad t \in[0,+\infty),
$$

in the case of more general than diagonal matrices $P_{i}(t)$, i.e. to obtain results on the exponential stability of the system

$$
X^{\prime \prime}(t)+\sum_{i=1}^{m} P_{i}(t) X\left(t-\tau_{i}(t)\right)=g(t), \quad t \in[0,+\infty) .
$$

5. To obtain results as regards the exponential stability of systems of functional differential equations of different orders.

\section{Competing interests}

The author declares that he has no competing interests.

Received: 23 May 2014 Accepted: 9 September 2014 Published: 24 Sep 2014

\section{References}

1. Azbelev, NV, Maksimov, VP, Rakhmatullina, LF: Introduction to Theory of Functional-Differential Equations. Nauka, Moscow (1991)

2. Brands, JJAM: Oscillation theorems for second-order functional-differential equations. J. Math. Anal. Appl. 63, 54-64 (1978)

3. Hale, JK: Theory of Functional Differential Equations. Springer, New York (1977)

4. Myshkis, AD: Linear Differential Equations with Delayed Argument. Nauka, Moscow (1972)

5. Domoshnitsky, A: Properties of Wronskian. In: Kraevye Zadachi, pp. 57-60. Politekh. Inst. Perm, Perm (1983) (in Russian)

6. Domoshnitsky, A: Unboundedness of solutions and instability of differential equations of the second order with delayed argument. Differ. Integral Equ. 14(5), 559-576 (2001)

7. Paatashvili, DV: About unbounded solutions of linear differential equations of second order with delay argument. Differ. Uravn. 25, 774-780 (1989)

8. Dolgii, YF, Nikolaev, SG: Instability of a periodic delay system. Differ. Uravn. 34, 463-468 (1998) 
9. Izjumova, DV: About boundedness and stability of solutions of nonlinear functional-differential equations of the second order. Proc. Georgian Acad. Sci. 100(2), 285-288 (1980) (in Russian)

10. Pinto, M: Nonlinear delay-differential equations with small lag. Int. J. Math. Math. Sci. 20, 137-146 (1997)

11. Pinto, M: Asymptotic solutions for second order delay differential equations. Nonlinear Anal. 28, 1729-1740 (1999)

12. Romanenko, EY, Sharkovskii, AN: Asymptotic Behavior of Solutions of Linear Functional-Differential Equations. Akad. Nauk of Ukraina, Inst. Math., Kiev (1978)

13. Derfel, GA, Molchanov, SA: On T. Kato's problem regarding bounded solutions of differential-functional equations. Funkc. Anal. Prilozh. 24, 67-69 (1990)

14. Dosla, Z, Kiguradze, IT: On boundedness and stability of solutions of second order linear differential equations with advanced arguments. Adv. Math. Sci. Appl. 9(1), 1-24 (1999)

15. Cahlon, B, Schmidt, D: Stability criteria for second-order delay differential equations with mixed coefficients. J. Comput. Appl. Math. 170, 79-102 (2004)

16. Cahlon, B, Schmidt, D: Stability criteria for certain second-order delay differential equations. Dyn. Contin. Discrete Impuls. Syst. 10, 593-621 (2003)

17. Burton, TA: Stability and Periodic Solutions of Ordinary Differential and Functional Differential Equations. Mathematics in Science and Engineering, vol. 178. Academic Press, San Diego (1985)

18. Burton, TA: Stability by Fixed Point Theory for Functional Differential Equations. Dover, New York (2006)

19. Domoshnitsky, A: About applicability of Chaplygin's theorem to one component of the solution vector. Differ. Uravn. 26, 1699-1705 (1990)

20. Bainov, D, Domoshnitsky, A: Stability of a second-order differential equations with retarded argument. Dyn. Stab. Syst. 9(2), 145-151 (1994)

21. Berezansky, L, Braverman, E, Domoshnitsky, A: Stability of the second order delay differential equations with a damping term. Differ. Equ. Dyn. Syst. 16(3), 185-205 (2008)

22. Pontryagin, LS: On zeros of some transcendental functions. Izv. Akad. Nauk SSSR, Ser. Mat. 6, 115-134 (1942) (the English translation in Am. Math. Soc. Transl., Ser. 2 1, 95-110 (1955))

23. Domoshnitsky, A: Maximum principles and nonoscillation intervals for first order Volterra functional differential equations. Dyn. Contin. Discrete Impuls. Syst., Ser. A Math. Anal. 15, 769-814 (2008)

24. Ladde, GS, Lakshmikantham, V, Zhang, BG: Oscillation Theory of Differential Equations with Deviating Argument. Dekker, New York (1987)

25. Norkin, SB: Differential Equations of the Second Order with Retarded Argument. Transl. Math. Monographs, vol. 31. Am. Math. Soc., Providence (1972)

26. Azbelev, NV: About zeros of solutions of linear differential equations of the second order with delayed argument. Differ. Uravn. 7(7), 1147-1157 (1971)

27. Koplatadze, RG: On oscillatory properties of solutions of functional differential equations. Mem. Differ. Equ. Math. Phys. 3, 1-179 (1994)

28. Agarwal, RP, Berezansky, L, Braverman, E, Domoshnitsky, A: Nonoscillation Theory of Functional Differential Equations with Applications. Springer, New York (2012)

10.1186/1029-242X-2014-361

Cite this article as: Domoshnitsky: Nonoscillation, maximum principles, and exponential stability of second order delay differential equations without damping term. Journal of Inequalities and Applications 2014, 2014:361

\section{Submit your manuscript to a SpringerOpen ${ }^{\circ}$ journal and benefit from:}

- Convenient online submission

Rigorous peer review

- Immediate publication on acceptance

Open access: articles freely available online

- High visibility within the field

- Retaining the copyright to your article 